\title{
Electrical current-driven dual-core optical fiber with embedded metal electrodes
}

\author{
Zhenggang Lian, Martha Segura, Nina Podoliak, Xian Feng, Nicolas White, Peter Horak, Wei H. Loh \\ Optoelectronics Research Centre, University of Southampton, SO17 1BJ, UK \\ z.lian@soton.ac.uk
}

\begin{abstract}
A dual suspended-core optical fiber with four embedded metal electrodes was fabricated by fiber drawing from a composite preform. Heating the fiber by running watt-level electrical power through the electrodes produced optical switching between cores. OCIS codes: (060.2280) Fiber design and fabrication; (060.4005) Microstructured fibers; (130.4815) Optical switching devices
\end{abstract}

\section{Introduction}

Single-material microstructured optical fibers have been developed with various structural designs, such as suspended core fibers [1] or photonic crystal fibers [2]; much of these efforts were focused on control of dispersion, enhancement of the nonlinearity or reduction of the fiber transmission loss. Fibers with metal or semiconductor insertions for electrical control have also been demonstrated: Through insertion of metal electrodes into a microstructured optical fiber polarization modulation was observed [3]. However, this post-processing is usually a time consuming approach and it limits the size of the devices. A metal-insulator-semiconductor fiber was demonstrated via a multi-material co-draw technique [4]. A fiber with a single internal electrode and an electrically conductive coating was fabricated by feeding a metal wire into the preform during fiber drawing [5].

A nanomechanical dual-core optical fiber was recently developed and optical switching between the two cores was demonstrated via pressure actuation [6]. In this fiber the cores are optically coupled through a small air gap. Mechanically moving one core alters the size of this air gap and thus the coupling length, which leads to the observed switching. Apart from pressurization, various other mechanisms can be envisaged to change the coupling length of such a dual-core fiber, e.g., electrostatic actuation [7], optomechanical forces, thermally changing the refractive index of the cores, etc.

Here we demonstrate the fabrication of a novel type of dual-core fiber and a novel actuation mechanism. Our fiber exhibits a dual suspended-core structure at the center and contains four metal electrodes embedded in the fiber cladding. The cores have dimensions of about $2.0 \times 3.1 \mu \mathrm{m}$, are separated by $200 \mathrm{~nm}$ of air and are suspended by two $500 \mathrm{~nm}$ thick glass membranes. Tens of meters of the fiber were fabricated with relatively uniform cores and metal electrodes. The temperature of the cores could be changed by applying electrical current to the electrodes and optical switching from one core to the other was observed by using a few hundred $\mathrm{mA}$ current.

\section{Fiber fabrication and characterization}

The fiber structure was based on lead-silicate glass (Schott F2) and low melting temperature tin. A dual-core preform was fabricated using the extrusion technique, and then drawn down to a smaller dimension to form the fiber cane. The dual-core cane was placed in the middle of a F2 jacket tube (10/8mm OD/ID) and surrounded by four glass tubes filled with tin (Fig. 1(a)). Four extra F2 rods were used to fill the gaps between the jacket and the tubes and to secure the position of the cane.

The multi-material dual-core fiber was drawn in two steps. Firstly, under nitrogen purging conditions, the preform was pre-heated to a temperature above the melting temperature of tin $\left(230^{\circ} \mathrm{C}\right)$ for 60 minutes in order to melt the tin and condense it down to the bottom of the preform. This step was found to be critical to minimize air bubbles between the metal and the glass, thus avoiding discontinuous wires in the fiber. Secondly, the preform was drawn into fiber using a drawing temperature of $730^{\circ} \mathrm{C}$ [4]. Fig. 1(b) shows an SEM picture of the fiber with the two suspended cores clearly seen in the center; a magnified picture is shown in Fig. 1(c). The size of the cores is $2.0 \times 3.1$ $\mu \mathrm{m}$, the membranes holding the cores are $12 \mu \mathrm{m}$ long and $500 \mathrm{~nm}$ thick. The average diameter of the four tin wires is $50 \mu \mathrm{m}$, and it was found that the wires are continuous over tens of meters by measuring the electrical resistance along the fiber. The optical loss of the fiber was measured for TE and TM polarizations as 2.5 and $4.8 \mathrm{~dB} / \mathrm{m}$, respectively. The loss mechanisms are expected to be the intrinsic loss of lead-silicate glasses $(1 \mathrm{~dB} / \mathrm{m}$ at $1.55 \mu \mathrm{m})$ and scattering due to surface roughness. 


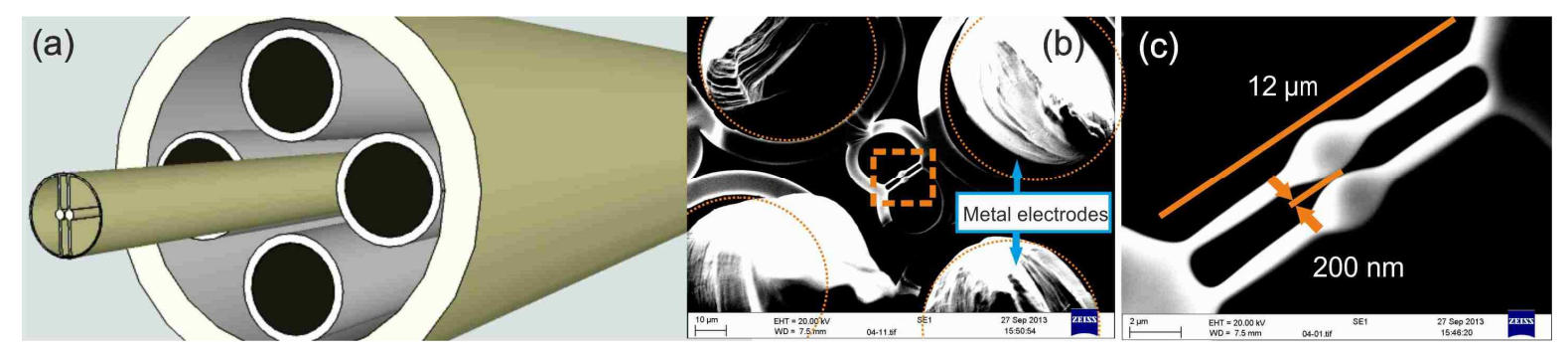

Fig. 1. (a) Schematic diagram of stacked preform of the metal embedded fiber; (b) SEM picture of the dual-core fiber with embedded metal electrodes and (c) detail of the cores in the yellow square of (b). The length of the membranes is 12 $\mu \mathrm{m}$, and the gap between two cores is $200 \mathrm{~nm}$.

To examine the electrical properties of the metal-embedded dual-core fiber, the glass part of the fiber was carefully cleaved and removed, leaving only the four electrodes for connecting purposes. The two ends of the metal embedded dual-core fiber were mounted, without bending or twisting the fiber, on two metallic V-grooves. The remaining portion of the fiber was coated with approximately $500 \mu \mathrm{m}$ thick acrylic to minimize instabilities due to variation in temperature and air turbulences surrounding the fiber during the experiment. The ends on the metallic V-grooves were left uncoated, thus allowing the V-grooves to act as heat sinks keeping the fiber temperature constant at the two fiber ends and allowing for stable launching conditions. The total length of the dual-core fiber was $65 \mathrm{~cm}$ with $50 \mathrm{~cm}$ coated with acrylic.

A diagram of the setup used for the optical characterization can be seen in Fig. 2. Collimated light from a 1550 $\mathrm{nm}$ wavelength pigtailed laser diode $(2 \mathrm{~mW})$ was focused into one of the cores with a microscope objective (L1) with 40x magnification and NA=0.65. The polarization state was TM (normal to the membranes) due to the higher contrast compared to that of TE (parallel to the membranes) and slightly stronger core-to-core coupling. At the output side, a 100x long working distance objective (L2) with NA=0.85 was used to image the output beam onto a CCD camera (SCOR-20-1550, Ophir-Spiricon Inc.).

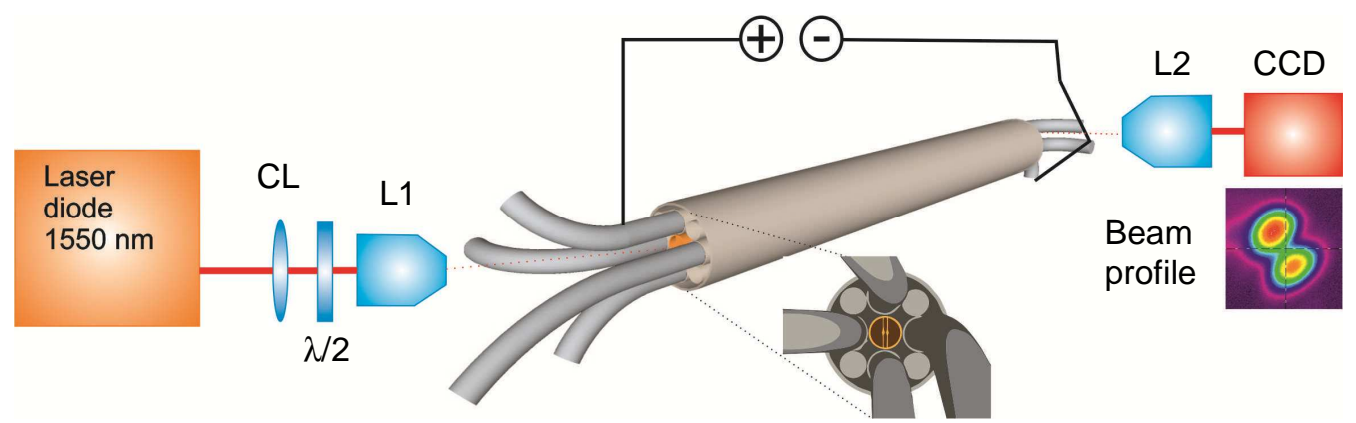

Fig. 2. Setup for optical characterization of the dual core fiber; CL is collimating lens, objective lens L1 and L2.

The four electrodes at each end were connected to the V-groove using silver paint and two copper wires coming from the power supply were soldered to each V-groove. In this way, one side of the metal electrodes was connected to the positive side of a power supply, and the other side of the metal electrodes was connected to the negative. The total resistance was measured and plotted in Fig. 3(a) where the ohmic response is clearly shown.

At room temperature, the guided light pattern shown on the CCD camera was stable. As the electric power increased, a noticable switching of light between the cores was observed. A sequence of light patterns was recorded consisting of 16 frames at 7.5 frames per second for each electric power level. The maximum intensity of light in each core was extracted from the 16 Gaussian profiles, averaged and normalized to the sum of intensities in both cores. The starting contrast was $0.55 / 0.45$ (top/bottom). As the electric power increased, a change in intensity of the light in each core was observed, see Fig. 3, reaching a maximum contrast of 0.38/0.62 at $0.4 \mathrm{~W}$ of electric power. The maximum applied electric power was $0.9 \mathrm{~W}$ where some instabilities in the output light pattern were observed. As shown in Fig. 3, an increase of power of $0.7 \mathrm{~W}$ was required to switch between the two points where both cores show the same output intensity. This behavior was repeatable, and the same results were observed when ramping the power up or down. We interpret the underlying mechanism for this observed switching as follows: By running an electric current through the electrodes, ohmic heating raises the fiber temperature. Because of the resulting thermal 
expansion of the fiber structure as well as the change of the glass refractive index, the optical coupling strength between the two cores is changed and subsequently also the output intensity pattern is modulated.
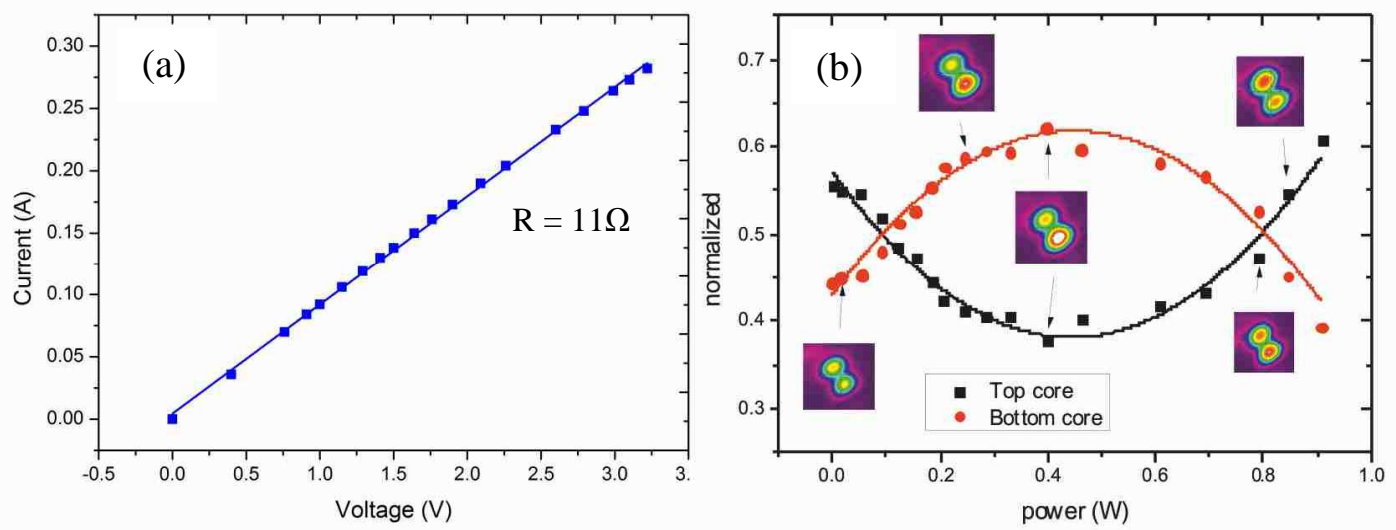

Fig. 3. (a) The total conductivity of the metal wire measured over $65 \mathrm{~cm}$; (b) Normalized output intensity in the upper (red dots) and lower (black squares) core as function of electric power. The intensity profiles shown were recorded at the CCD camera. The solid lines are sinusoidal fits to the experimental data.

In order to confirm this interpretation, we numerically modeled the fiber using a finite element method (Comsol Multiphysics). The physical dimensions of the fiber cores, the membranes and the electrodes were obtained from the SEM photos (Fig. 1(b) and 1(c)) and the optical modes supported by the fiber were simulated as a function of temperature including the effects of thermal expansion in the longitudinal and perpendicular directions (expansion coefficient of $\mathrm{F} 2$ glass $\left.\alpha=9.2 \times 10^{-6} \mathrm{~K}^{-1}\right)$ and temperature-dependent refractive index $\left(\mathrm{dn} / \mathrm{dT}=2.7 \times 10^{-6} \mathrm{~K}^{-1}\right)$. These simulations predict a required temperature change of $7.85 \mathrm{~K}$ to achieve switching from one core to the other for the fundamental mode with TM polarization, while for the next higher order mode it is $21.35 \mathrm{~K}$.

In a second model, we investigated the heat transfer and stationary temperature distribution in our fiber structure where the current-carrying electrodes act as heating elements. If the temperature of the outer surface of the acrylic coating is kept fixed at room temperature (tantamount to assuming an ideal heat sink), $1 \mathrm{~W}$ of electrical power increases the temperature of the cores by $2 \mathrm{~K}$ with respect to room temperature. Allowing for an additional thin ( 500 $\mu \mathrm{m}$ ) layer of air insulating the fiber further increases the core temperature to $\sim 10 \mathrm{~K}$ above room temperature at the same $1 \mathrm{~W}$ electric power, which according to the simulations discussed above leads to switching of light between the cores. This latter scenario is thus in good agreement with the experimental observations. We are still working on the fabrication of improved versions of our metal-embedded dual-core fiber that would allow us to observe one full switching cycle.

\section{Conclusion}

We have described the fabrication of a dual-core optical fiber with embedded metal electrodes for novel fiber based devices. Optical switching was demonstrated by driving electrical current through the electrodes and thus changing the temperature of the cores. These results show the potential of such fibers in optical networking applications.

We gratefully acknowledge support for this work from the Engineering and Physical Sciences Research Council (EPSRC) through funding for the EPSRC Centre for Innovative Manufacturing in Photonics, the "Photonic Hyperhighway" Programme Grant, and the "Tunable Optical Buffer" grant (EP/J012874/1).

\section{References}

[1] P. Kaiser, E.A.J. Marcatili, and S.E. Miller, “A new optical fiber,” Bell System Technical Journal 52(2), 265-169 (1973).

[2] P. Russell, "Photonic crystal fibre," Science 299(5605), 358-362 (2003).

[3] G. Chesini, C. M. B. Cordeiro, C. J. S. de Matos, M. Fokine, I. C. S. Carvalho, and J. C. Knight, "All-fiber devices based on photonic crystal fibers with integrated electrodes," Opt. Express 17(3), 1660-1665 (2009).

[4] M. Bayindir, F. Sorin, A.F. Abouraddy, J. Viens, S.D. Hart, J.D. Joannopoulos, and Y. Fink, "Metal-insulator-semiconductor optoelectronic fibres," Nature 431(7010), 826-829 (2004).

[5] K. Lee, P. Henry, F. Simon, and J.L. Blows, "Drawing of optical fiber with internal codrawn wire and conductive coating and electrooptic modulation demonstration," Photonics Technology Letters, IEEE 18(8), 914-916 (2006).

[6] Z. Lian, P. Horak, X. Feng, L. Xiao, K. Frampton, N. White, J. Tucknott, H. Rutt, D.N. Payne, W. Stewart, and W.H. Loh, "Nanomechanical optical fiber," Opt. Express 20(28), 29386-29394 (2012).

[7] N. Podoliak, Z. Lian, P. Horak, and W.H. Loh. Electrostatic control of dual-core optical fibre with NEMS functionality. in CLEO-

Europe/IQEC 2013. Munich, 2013. 